\title{
Disentangling genetic variation for resistance and tolerance to infectious
}

\section{diseases in animals}

Lars Råberg ${ }^{1,2^{*}}$, Derek $\operatorname{Sim}^{1}$ and Andrew F. Read ${ }^{1 \dagger}$

${ }^{1}$ Institutes of Evolutionary Biology \& Immunology and Infection Research, School of Biological Sciences, University of Edinburgh, West Mains Road, Edinburgh EH9 3JT, $\mathrm{UK}$

${ }^{2}$ Department of Animal Ecology, Lund University, Ecology Building, 22362 Lund, Sweden

* Correspondence, present address: Department of Animal Ecology, Lund University, Ecology Building, 22362 Lund, Sweden, lars.raberg@zooekol.lu.se $†$ Present address: Center for Infectious Disease Dynamics, The Pennsylvania State University, 208 Mueller Lab, University Park, P.A. 16802, U.S.A. 
Science 2 November 2007: Vol. 318. no. 5851, pp. 812 - 814

DOI: $10.1126 /$ science. 1148526

\section{Abstract}

Hosts can in principle employ two different strategies to defend themselves against parasites: resistance and tolerance. Animals typically exhibit considerable genetic variation for resistance (the ability to limit parasite burden). However, little is known about whether animals can evolve tolerance (the ability to limit the damage caused by a given parasite burden). Using rodent malaria in laboratory mice as a model system, and the statistical framework developed by plant-pathogen biologists, we demonstrated genetic variation for tolerance, as measured by the extent to which anemia and weight loss increase with increasing parasite burden. Moreover, resistance and tolerance were negatively genetically correlated. These results mean that animals, like plants, can evolve two conceptually different types of defense, a finding that has important implications for the understanding of the epidemiology and evolution of infectious diseases. 
Defense against pathogenic micro-organisms and other parasites can be divided into two conceptually different components: resistance (the ability to limit parasite burden) and tolerance (the ability to limit the disease severity induced by a given parasite burden)( 1 4). It is important to distinguish between these two components because, by definition, resistance has a negative effect on parasites while tolerance does not; as a result, their relative importance will have significant consequences for the ecology and evolution of host-parasite interactions $(2,5-7)$. The distinction between resistance and tolerance has attracted considerable attention in studies of the evolution of plant defense against parasites and herbivores $(3,6,8)$. Here, the emerging pattern is that plants generally exhibit genetic variation for both resistance and tolerance $(1,6,9,10)$.

When it comes to animals, numerous studies have demonstrated genetic variation for resistance, where resistance is typically measured as the inverse of parasite burden (e.g. refs 11-14). However, little is known about whether animals also may show genetic variation for tolerance. Yet together resistance and tolerance are the two components of anti-pathogen defense that determine disease severity. Suggestive evidence for tolerance in animals comes for example from a study of $\alpha^{+}$-thalassemia, a monogenic hemoglobin disorder in humans that protects against malaria. Individuals hetero- or homozygous for this mutation do not have lower infection intensities of $P$. falciparum than individuals homozygous for the wild type, but the degree of anemia at high infection intensities is diminished, thereby reducing mortality from malaria (15). Thus, it seems $\alpha^{+}$-thalassemia affects tolerance but not resistance to $P$. falciparum. So far as we are aware, no study has 
yet formally disentangled genetic variation in these two components of defense in any animal host-parasite system.

In the plant literature, tolerance is usually defined as the slope of host fitness against infection intensity $(1,16,17)$. In other words, the tolerance of a host genotype is its reaction norm to infection intensity. A tolerant genotype is one in which disease severity is relatively unaffected by increasing pathogen burden, whereas the fitness of a less tolerant genotype more rapidly declines as pathogen burdens rise (Fig 1). If the reaction norms of different host genotypes differ (i.e., if there is a statistical interaction between host genotype and infection intensity), then there is genetic variation for tolerance. We have borrowed this approach to defining and measuring genetic variation in tolerance from the plant literature and applied it to a malaria model systemPlasmodium chabaudi in laboratory mice — to investigate whether animal hosts may show genetic variation for tolerance, and whether resistance and tolerance are correlated traits.

Plasmodium chabaudi is widely used as a model of human malaria $(18,19)$. Previous studies have shown that there is considerable variation among mouse strains (i.e., genetic variation) for resistance to P. chabaudi (20-22). To investigate whether there is also genetic variation for tolerance, we performed an experiment with five different inbred mouse strains. Mice were infected with one of three different $P$. chabaudi clones or left uninfected in a fully factorial design. The experiment was performed in three experimental blocks separated in time. As with human malaria, one of the main causes of morbidity and mortality in rodent malaria is anemia. Plasmodium chabaudi also causes 
Science 2 November 2007: Vol. 318. no. 5851, pp. 812 - 814

DOI: $10.1126 /$ science. 1148526

weight loss in mice. The degree of red blood cell (RBC) loss and weight loss is correlated with infection intensity and predicts mortality (23). To test for variation for tolerance, we therefore used minimum RBC density and minimum weight during the infection as measures of disease severity (analogous to host fitness used in the plant literature). Specifically, we tested whether the slopes of the relationship between infection intensity and minimum RBC density or minimum weight differed between mouse strains.

As usual in this host-parasite system $(24,25)$, there was a distinct peak in parasite density around day 8 post inoculation. Minimum RBC density occurred around day 11, on average $2.49 \pm 0.88$ (mean \pm s.d.) days after peak parasite density. Minimum weight occurred around day 10-11, on average $1.60 \pm 2.89$ days after peak parasite density (Fig S1).

To test for variation for resistance among mouse strains, we performed an analysis of peak parasite density against mouse strain and parasite clone. Peak parasite density differed between mouse strains $[F(4,102)=15.54, P<0.0001]$ and parasite clones $[F(2$, $103)=64.81, P<0.0001]$, but there was no strain-by-clone interaction $[F(8,102)=0.66$, $P=0.73]$. There was also a significant effect of experimental block $\left(\chi^{2}=47.4, \mathrm{P}<0.0001\right)$, but no interactions between block and strain and/or clone $(\mathrm{P}>0.25)$. Thus, as in previous studies $(20-22,24)$, mouse strains differed in resistance, and parasite clones differed in the infection intensity they induced. 
Science 2 November 2007: Vol. 318. no. 5851, pp. 812 - 814

DOI: $10.1126 /$ science. 1148526

To test for genetic variation for tolerance, we performed analyses of minimum RBC density and minimum weight against peak parasite density (both linear and quadratic terms), mouse strain, their interactions, and pre-inoculation values of RBC density or weight. In the case of both RBC and weight loss, there were highly significant interactions between strain and parasite density (Fig 2). Thus, there was variation among mouse strains in tolerance measured in terms of either anemia or weight loss. This conclusion is robust to the inclusion of parasite clone in the statistical models, the exclusion of uninfected animals from the analyses, or the use of different infection intensity measures (see supporting online text).

To test whether these two estimates of tolerance were correlated we calculated the slopes of minimum weight and RBC density against peak parasite density for each mouse strain. There was a significant correlation between the two measures of tolerance (Spearman's rank correlation, $\mathrm{r}_{\mathrm{s}}=1.0, \mathrm{n}=5, P<0.05$ ). Importantly, there were also significant negative correlations between resistance and both measures of tolerance $\left(\mathrm{r}_{\mathrm{s}}=-1.0, \mathrm{n}=5, P<0.05\right.$ in the case of both RBC loss and weight loss; Fig 3). DBA mice, for example, were more tolerant and less resistant than $\mathrm{C} 57 \mathrm{~s}$, which were the opposite. Thus, reduced tolerance is a cost of resistance and vice versa.

Our studies demonstrate that the conceptual and analytical framework developed by plant evolutionary biologists can also be used with animals to reveal genetic variation for tolerance to infectious diseases. The existence of genetic variation for both resistance and tolerance means that host defense can take a variety of evolutionary trajectories in 
response to pathogen pressure. The mechanistic basis of the genetic variation in tolerance we report remains to be determined. Variation in tolerance measured as RBC loss could be due either to that the rate of regeneration of $\mathrm{RBC}$, or that the rate of destruction of RBC by parasites and/or host immune responses, varies among strains. The correlation between tolerance measured as RBC and weight loss suggests that there is a common underlying factor between these two forms of tolerance. In plants, where tolerance has long been studied, genes conferring disease tolerance have yet to be identified at the molecular level (6). In our disease model, resistance and tolerance were traded off against each other (Fig 3). A similar trade-off has previously been demonstrated in the context of plant defense against herbivory (2). In our case, the trade-off could arise if the price of more aggressive immune control of infection is increasing collateral damage (immunopathology).

Our findings, if they prove general, have important implications for our understanding of the ecology and evolution of animal host-parasite interactions. First, while the evolution of resistance has a negative effect on the prevalence of the infectious agent in the host population, tolerance should have a neutral or positive effect. Thus, resistance and tolerance have contrasting effects on the epidemiology of infectious diseases $(5,7)$. Second, hosts and parasites are commonly thought to be engaged in antagonistic coevolution, where evolution of host resistance selects for counteradaptations in the parasite, which selects for improved resistance in the host and so on, leading to open-ended non-equilibrium evolutionary dynamics (26). However, tolerance does not have a negative effect on the fitness of the parasite, and so it cannot fuel 
antagonistic coevolution in the same way as resistance is expected to do. Genetic variation for tolerance can therefore be expected to allow the sort of host evolution that will substantially dampen antagonistic coevolution (6).

Beyond evolutionary ecology, there is a clear need to recognise and separate the two components of disease defense in the context of animal breeding. For instance, attempts to enhance yield in agricultural animals by artificial selection on disease resistance traits, or on total yield in the face of infection [often referred to as "resilience"(27)], could generate a variety of more or less desirable outcomes, depending on how resistance or yield vary with tolerance. The experimental and analytic approach used here is readily transferable to domestic animals where it could be used to work out optimal selection strategies.

\section{References and Notes}

1. E. L. Simms, J. Triplett, Evolution 48, 1973 (1994).

2. W. Fineblum, M. Rausher, Nature 377, 517 (1995).

3. J. Fornoni, J. Nuñez-Farfan, P. L. Valverde, M. Rausher, Evolution 58, 1685 (2004).

4. O. Restif, J. C. Koella, American Naturalist 164, E90 (2004).

5. B. A. Roy, J. W. Kirchner, Evolution 54, 51 (2000). 
Science 2 November 2007: Vol. 318. no. 5851, pp. 812 - 814

DOI: $10.1126 /$ science. 1148526

6. M. D. Rausher, Nature 411, 857 (2001).

7. M. R. Miller, A. White, M. Boots, Evolution 60, 945 (2006).

8. K. Stowe, R. Marquis, C. Hochwender, E. L. Simms, Annual Review of Ecology and Systematics 31, 565 (2000).

9. P. X. Kover, B. A. Schaal, Proceedings of the National Academy of Sciences of the United States of America 99, 11270 (2002).

10. T. Koskela, S. Puustinen, V. Salonen, P. Mutikainen, Evolution 56, 899 (2002).

11. D. Malo, E. Skamene, Trends in Genetics 10, 365 (1994).

12. A. V. S. Hill, Annual Review of Immunology 16, 593 (1998).

13. J. A. Smith, K. Wilson, J. G. Pilkington, J. M. Pemberton, Proceedings of the Royal Society of London, B 266, 1283 (1999).

14. T. N. Williams et al., Nature Genetics 37, 1253 (2005).

15. S. Wambua et al., PLOS Medicine 3, 0643 (2006).

16. P. Tiffin, M. D. Rausher, American Naturalist 154, 700 (1999).

17. E. L. Simms, Evolutionary Ecology 14, 563 (2000).

18. M. M. Stevenson, E. M. Riley, Nature Reviews Immunology 4, 169 (2004).

19. A. Martinelli et al., Proceedings of the National Academy of Sciences of the United States of America 102, 814 (2005). 
20. M. M. Stevenson, J. J. Lyanga, E. Skamene, Infection and Immunity 38, 80 (1982).

21. A. Fortin, M. M. Stevenson, P. Gros, Genes and Immunity 3, 177 (2002).

22. K. Grech, K. Watt, A. F. Read, Journal of Evolutionary Biology 19, 1620 (2006).

23. M. J. Mackinnon, A. F. Read, Philosophical Transactions of the Royal Society of London B 359, 965 (2004).

24. M. J. Mackinnon, A. F. Read, Evolution 53, 689 (1999).

25. J. C. De Roode et al., Proceedings of the National Academy of Sciences of the United States of America 102, 7624 (2005).

26. M. E. J. Woolhouse, J. P. Webster, E. Domingo, B. Charlesworth, B. Levin, Nature Genetics 32, 569 (2002).

27. S. A. Bisset, C. A. Morris, International Journal for Parasitology 26, 857 (1996).

28. We thank Andrea Graham, Henrik Smith and Martin Stjernman for extensive discussion. This work was funded by the Wellcome Trust (to AFR), a Marie Curie fellowship (to LR; FP6-501567) and the Swedish Research Council (to LR). This paper was written while AR was at the Wissenschaftskolleg zu Berlin. The experimental work was conducted on Project Licence PPL 60/2714 granted by the Home Office under the auspices of the United Kingdom Animals (Scientific Procedures) Act of 1986.

\section{Supporting Online Material}


Science 2 November 2007: Vol. 318. no. 5851, pp. 812 - 814

DOI: $10.1126 /$ science. 1148526

WWW.sciencemag.org

Material and Methods

Supporting online text

Fig S1

References 
Science 2 November 2007: Vol. 318. no. 5851, pp. 812 - 814

DOI: $10.1126 /$ science. 1148526

Fig 1. Schematic figure showing reaction norms of two host genotypes (red or blue line) for disease severity across a range of infection intensities in individual hosts (dots). (A) Two equally tolerant genotypes differing in resistance; here, the red genotype has lower parasite burdens (is more resistant) and thereby maintains higher health status when infected. (B) Two equally resistant genotypes (same parasite burden) but here the red genotype is less tolerant (health declines faster with increasing parasite burden). (C) Genotypes differ in both tolerance and resistance; here, the more tolerant genotype (blue) is less resistant, so that both genotypes end up having on average the same health status when infected. (D) Host genotypes differ in neither resistance (same average parasite burden) nor tolerance (same slopes). Instead, the genetic difference in health status is due to a difference in intercept, so that the difference exists even when animals are uninfected. It is thus indicative of genetic differences in "general vigor"( 8$)$, and has nothing to do with defense against the infectious agent in question. Because of the possible existence of variation in general vigor, tolerance has to be defined as a reaction norm, and so it can only be measured and compared across groups of animals (17). Thus, in contrast to resistance, it is not possible to compare the tolerance of two individual hosts. Demonstrating genetic variation for tolerance therefore requires that disease severity be assessed in animals of the same genotype across a range of infection intensities; a difference in slope between genotypes indicates genetic variation for tolerance.

Fig 2. Variation for tolerance among mouse strains. (A) Minimum RBC density (logtransformed) against peak parasite density. Mouse strain $\times$ parasite density: $F(4$, 
$117)=6.08, P=0.0002$; parasite density: $F(1,117)=173.3, P<0.0001$; mouse strain $F(4$, 117) $=0.20, P=0.94$; experimental block: $\chi^{2}=22.1, P<0.0001$. Initial RBC density $[F(1$, 116) $=0.80, P=0.37$ ], the quadratic terms [parasite density ${ }^{2}: F(1,117)=0.76, P=0.38$; strain $\times$ parasite density $\left.{ }^{2}: F(4,111)=0.33, P=0.86\right]$, and the interaction between block and strain $(P>0.25)$ were not significant and therefore excluded from the model. (B) Minimum weight (log-transformed) against peak parasite density. Strain×parasite density: $F(4,110)=6.06, P=0.0002$; parasite density: $F(1,111)=8.09, P=0.0053$; parasite density $^{2}: F(1,111)=34.4, P<0.0001$; mouse strain: $F(4,110)=2.76, P=0.031$; initial weight: $F(1,111)=140, P<0.0001$; experimental block: $\chi^{2}=18.1, \mathrm{P}<0.0001$. Strain $\times$ parasite density ${ }^{2}[F(4,105)=1.20, P=0.31]$ and the interaction between block and strain $(P>0.25)$ were not significant, and these terms were therefore excluded. To facilitate comparison of slopes, and because initial weight (the intercept) differed between strains but for the present purposes is an irrelevant main effect when testing for resistance and tolerance, the reaction norms for weight have been scaled so that all genotypes have an intercept of 0 .

Fig 3. Trade-off between tolerance and resistance. (A) Correlation between resistance (inverse of peak parasite density) and tolerance in the form of minimum RBC density during infection (i.e., tolerance measured as slope of regression of minimum RBC density against peak parasite). (B) Correlation between resistance and tolerance in the form of minimum weight during infection (i.e., tolerance measured as slope of regression of minimum weight against peak parasite density). Plots show mean \pm s.e. for each mouse strain. 
Science 2 November 2007: Vol. 318. no. 5851, pp. 812 - 814

DOI: $10.1126 /$ science. 1148526

Fig 1 Råberg et al.

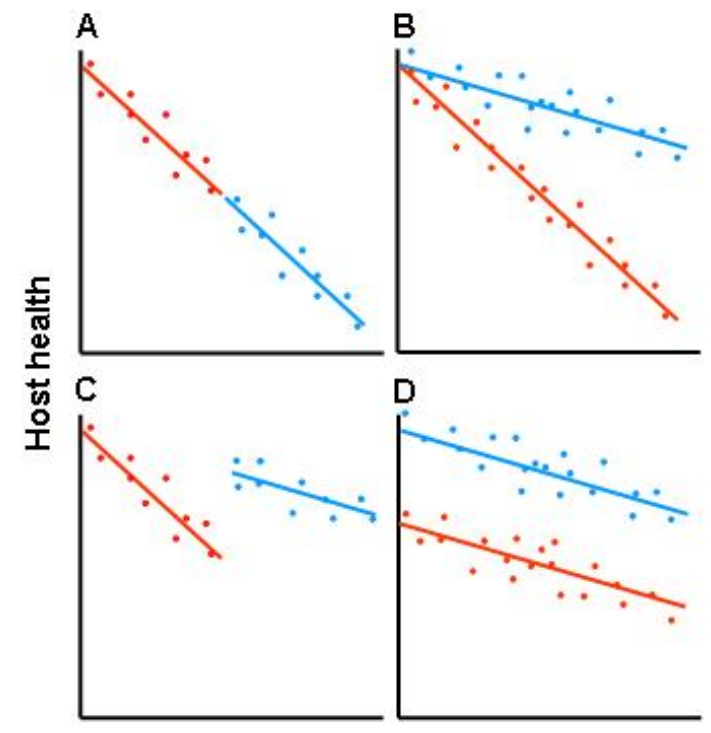

Parasite burden 
Science 2 November 2007: Vol. 318. no. 5851, pp. 812 - 814

DOI: $10.1126 /$ science. 1148526

Fig 2 Răberg et al.
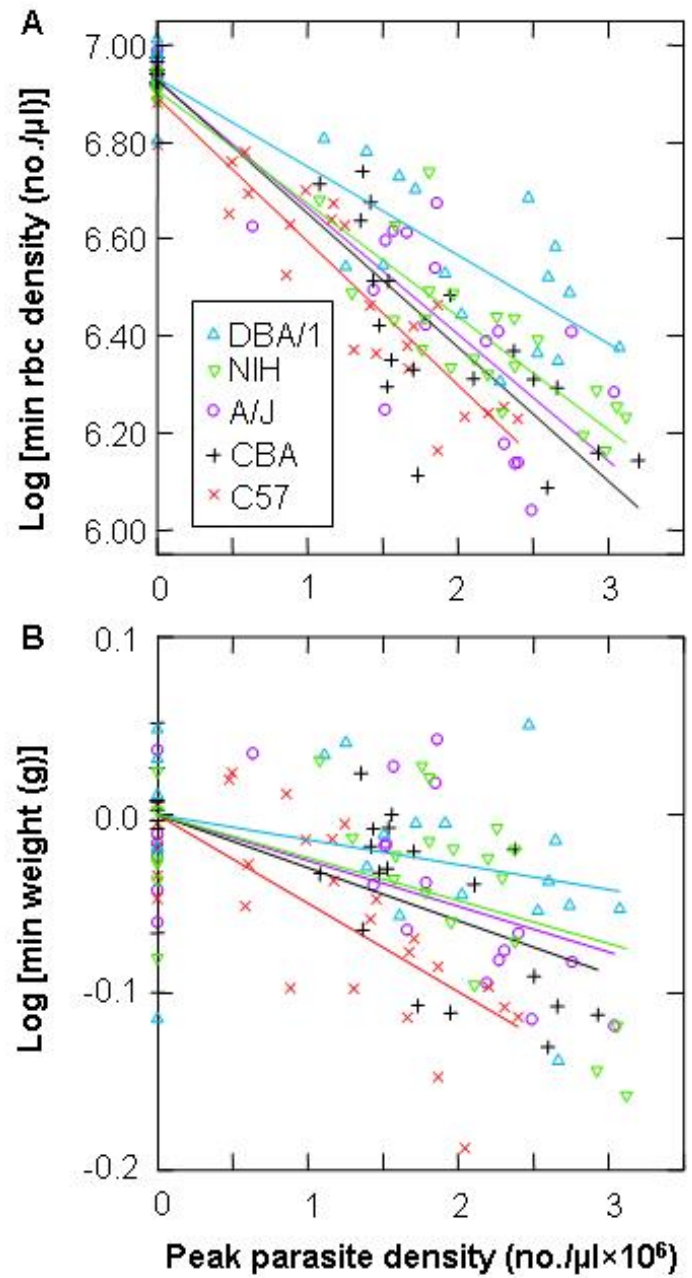
Science 2 November 2007: Vol. 318. no. 5851, pp. 812 - 814

DOI: $10.1126 /$ science. 1148526

Fig 3 Răberg et al.
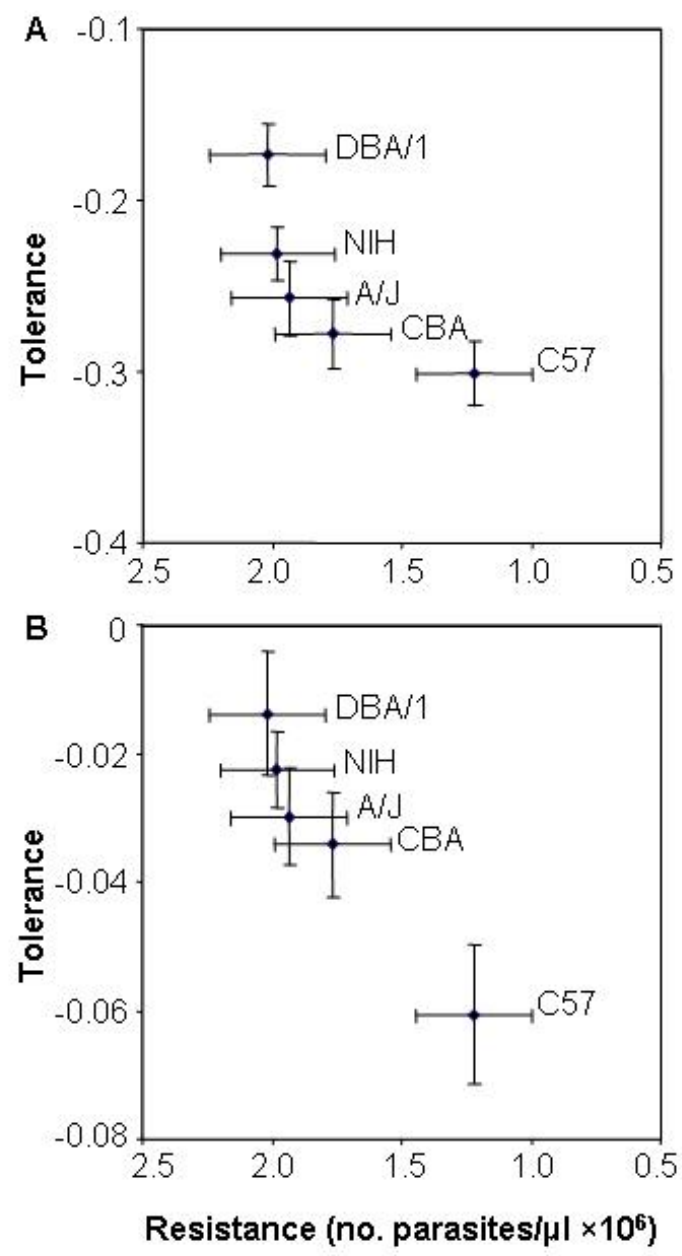
Science 2 November 2007: Vol. 318. no. 5851, pp. 812 - 814

DOI: $10.1126 /$ science. 1148526

\section{Supporting Online Material}

\section{Materials and Methods}

Host and parasite

We used five strains of inbred mice: A/J, C57BL/6J, CBA/Ca, DBA/1 and NIH (Harlan, UK). Strains were chosen based on previous work $(1,2)$ to include both relatively resistant and non-resistant strains. All mice were 9-10 weeks old at the start of the experiment. We used three different parasite clones, denoted $\mathrm{AS}_{11849}, \mathrm{AJ}_{4777}$ and $\mathrm{DK}_{104}$. Clones were selected based on previous studies $(3,4)$, to maximize variation in infection intensity.

\section{Setup and sampling}

Each mouse was infected with one of the three parasite clones or left uninfected. The inoculation dose was $10^{5}$ parasites. Inoculations were performed as described by de Roode et al. (3). The experiment was performed in three experimental blocks. In total the experiment comprised 152 mice ( $\mathrm{N}=29-32$ of each strain).

We weighed mice on an electronic balance and took blood samples from the tail before inoculation and then daily for days 5-15 post inoculation (p.i.) to measure infection intensity and RBC density. We use maximum parasite density (no. of parasites $/ \mu$ blood) as a measure of infection intensity. Another common measure of infection intensity in the malaria literature is the maximum proportion of infected RBC. These two measures are strongly correlated ( $\mathrm{r}=0.87$ in the present data set) and analyses based on parasite density and proportion infected RBC yield the same conclusions. We 
Science 2 November 2007: Vol. 318. no. 5851, pp. 812 - 814

DOI: $10.1126 /$ science. 1148526

measured RBC density using flow cytometry (Beckman Coulter) and estimated the proportion of infected RBC by microscopy; parasite density was calculated by multiplying these values.

\section{Data set and statistical analyses}

We analyzed the data by means of mixed linear models. Mouse strain and parasite clone were treated as fixed effects, while experimental block and its interactions with strain and clone were treated as random effects. The significance of random effects was assessed by log-likelihood ratio test (5). Non-significant random effects were excluded from the model at P>0.25. Analyses were performed with PROC MIXED in SAS 9.1 (6), using the Satterthwaite approximation of denominator df of fixed effects.

In analyses testing for variation in tolerance, we used $\log$ (minimum RBC density) or $\log ($ minimum weight $)$ as dependent variables, and $\log$ (pre inoculation value) as covariate (if statistically significant). The variables were log-transformed because we wanted to test for proportionate changes in minimum weight and RBC density with increasing infection intensity.

If the relationship between disease severity (here minimum RBC density and minimum weight) and infection intensity is nonlinear, but only linear terms are included in the statistical model, this may give rise to spurious variation in tolerance (7). We therefore tested for non-linear relationships by including quadratic terms in the models. Slopes were estimated with Z-transformed data (i.e., mean=0, s.d.=1).

Twenty five per cent of the infected mice died or were euthanized, all between day 10 and 14 . The mortality presents a potential problem for the analysis of tolerance 
because in mice that died, minimum weight and RBC density often occurred on the day of death. To ensure that the results were not biased by mice that died before reaching even lower values, unambiguous minima were obtained by including in the analyses of tolerance only mice which had survived long enough for their RBC density/weight to begin to increase again ( $\mathrm{N}=129$ for minimum $\mathrm{RBC}$ and $\mathrm{N}=123$ for minimum weight) [(for the sake of completeness, we also the present analyses based on all mice in the supporting online text (see below)]. However, analyses of resistance were based on all mice, because mice that died had in all cases passed the peak parasite density.

\section{Supporting text}

\section{The inclusion of clone in the statistical models}

In the analyses of tolerance above we assume that the severity of disease induced by a particular parasite genotype (the RBC or weight loss it causes) is a direct consequence of its infection intensity, and that there is no difference in per parasite virulence between clones. The same assumption is made in previous studies of tolerance in plants [which have used parasites of unknown genetic composition, e.g. refs (8-10)]. However, the per parasite virulence could differ between parasite genotypes. We therefore repeated the analysis of tolerance including also the factor clone and its interactions (in this analysis we excluded uninfected mice; thus, the factor clone has 3 levels: DK, AS or AJ; N=96 and 90 for minimum RBC density and minimum weight, respectively). In the case of minimum RBC density, there were significant effects of both clone $[F(2,76)=92.9$, $P<0.0001]$ and strain $\times$ clone $[F(8,76)=3.61, P=0.0013]$. However, the tolerance term (strain $\times$ infection intensity) remains significant when controlling for these effects $[F(4$, 
76) $=4.75, P=0.0018]$. Also in the case of minimum weight there was an effect of clone $[F(2,77)=29.5, P<0.0001]$, but again the strain $\times$ infection intensity term remained significant $[F(4,77)=2.69, P=0.037]$. Thus, variation for tolerance is not confounded by clonal variation in per parasite virulence. This analysis also shows that the variation for tolerance we report is not arising as some artefactual consequence of including the uninfected mice.

The use of parasite intensity measures other than peak density Variation in infection intensity may not be fully captured by peak density. For example, the rate at which the infection intensity declines after the peak may also affect anaemia and weight loss. If mouse strains differ with respect to such infection dynamics, this may result in spurious variation for tolerance. Therefore, we repeated the analyses of tolerance using the total number of parasites present in an infection as measure of infection intensity. For these analyses, we selected mice that survived at least 3 days post peak and calculated total densities by summing the daily densities (the generation time for the asexual stage of $P$. chabaudi is $24 \mathrm{~h}$ ) from day 2 pre peak up to and including day 3 post peak ( $\mathrm{N}=112$ for minimum $\mathrm{RBC}$ density and $\mathrm{N}=99$ for minimum weight). Analyses of both minimum RBC density and minimum weight using this measure of infection intensity yielded the same conclusions as the analyses with peak density above (min RBC density: initial RBC density: $F(1,101)=9.63, P=0.002$; total parasite density: $\mathrm{F}(1$, 99.2) $=192, P<0.0001$; strain: $F(4,99.5)=0.59, P=0.67$; density $\times$ strain: $F(4,99.3)=6.76$, $P<0.0001$; experimental block: $\chi^{2}=27.9, P<0.0001$; block $\times$ strain: $P>0.25$; minimum weight: initial weight: $F(1,83)=105, \mathrm{P}<0.0001$, strain: $F(4,83)=7.80, P<0.0001$; density, 
linear term: $F(1,83)=70.8, P<0.0001$; density, quadratic term: $F(1,83)=35.8, P<0.0001$; density $\times$ strain: $F(4,83)=4.23, P=0.004 ;$ strain $\times$ density $\left.^{2}: F(4,83)=2.54, P=0.046\right)$; experimental block and strain $\times$ block: $P>0.25$. Thus, there is no reason to suspect that the strain-by-infection intensity interactions are particular to the measure of parasite burden.

\section{Analyses based on all mice}

As described in the Materials and Methods above, the main analyses of tolerance (fig 2) are based on a subset of data. Specifically, we excluded mice whose RBC density and/or weight did not start to rise before they died. However, the exclusion of these mice could possibly bias the results, if mice that died before reaching minimum $\mathrm{RBC}$ density/weight are not random with respect to tolerance. We therefore also performed analyses based on all mice $(\mathrm{N}=152)$. These analyses yielded the same conclusions as the analyses presented in fig 2: Minimum RBC density: Strain: $F(4,140)=0.26, P=0.90$; peak parasite density: $F(1,140)=147.4, P<0.0001$; strain $\times$ density: $F(4,140)=5.61, P=0.0003$; experimental block: $\chi^{2}=19.1, P<0.0001$. Initial RBC density $(P=0.49)$, parasite density ${ }^{2}(P=0.20)$ and block $\times$ strain $(P>0.25)$ were not significant and therefore excluded from the model. Minimum weight: initial weight: $F(1,140)=177.0, P<0.0001$; strain: $F(4,139)=1.92$, $P=0.11$; peak parasite density: $F(1,140)=3.54, P=0.062$; density ${ }^{2}: F(1,140)=25.0$, $P<0.0001$; strain $\times$ density: $F(4,138)=3.99, P=0.0043$; experimental block: $\chi^{2}=22.2$, $P<0.0001$. Strain $\times$ density $^{2}(P=0.44)$ and block $\times$ strain $(P>0.25)$ were not significant and therefore excluded from the model.

Reference List 
Science 2 November 2007: Vol. 318. no. 5851, pp. 812 - 814

DOI: $10.1126 /$ science. 1148526

1. A. Fortin, M. M. Stevenson, P. Gros, Genes and Immunity 3, 177 (2002).

2. K. Grech, K. Watt, A. F. Read, Journal of Evolutionary Biology 19, 1620 (2006).

3. J. C. De Roode, R. Culleton, S. J. Cheesman, R. Carter, A. F. Read, Proceedings of the Royal Society of London, B 271, 1073 (2004).

4. K. Grech, PhD thesis, University of Edinburgh, UK (2006).

5. G. P. Quinn, M. J. Keough, Experimental design and data analysis for biologists (Cambridge University Press, Cambridge, 2002).

6. SAS Institute, SAS OnlineDoc, V8 (SAS Institute, Cary, USA, 1999).

7. P. Tiffin, B. D. Inouye, Evolution 54, 1024 (2000).

8. E. L. Simms, J. Triplett, Evolution 48, 1973 (1994).

9. P. X. Kover, B. A. Schaal, Proceedings of the National Academy of Sciences of the United States of America 99, 11270 (2002).

10. T. Koskela, S. Puustinen, V. Salonen, P. Mutikainen, Evolution 56, 899 (2002). 
Science 2 November 2007: Vol. 318. no. 5851, pp. 812 - 814

DOI: $10.1126 /$ science. 1148526

Fig S1 Răberg et al.
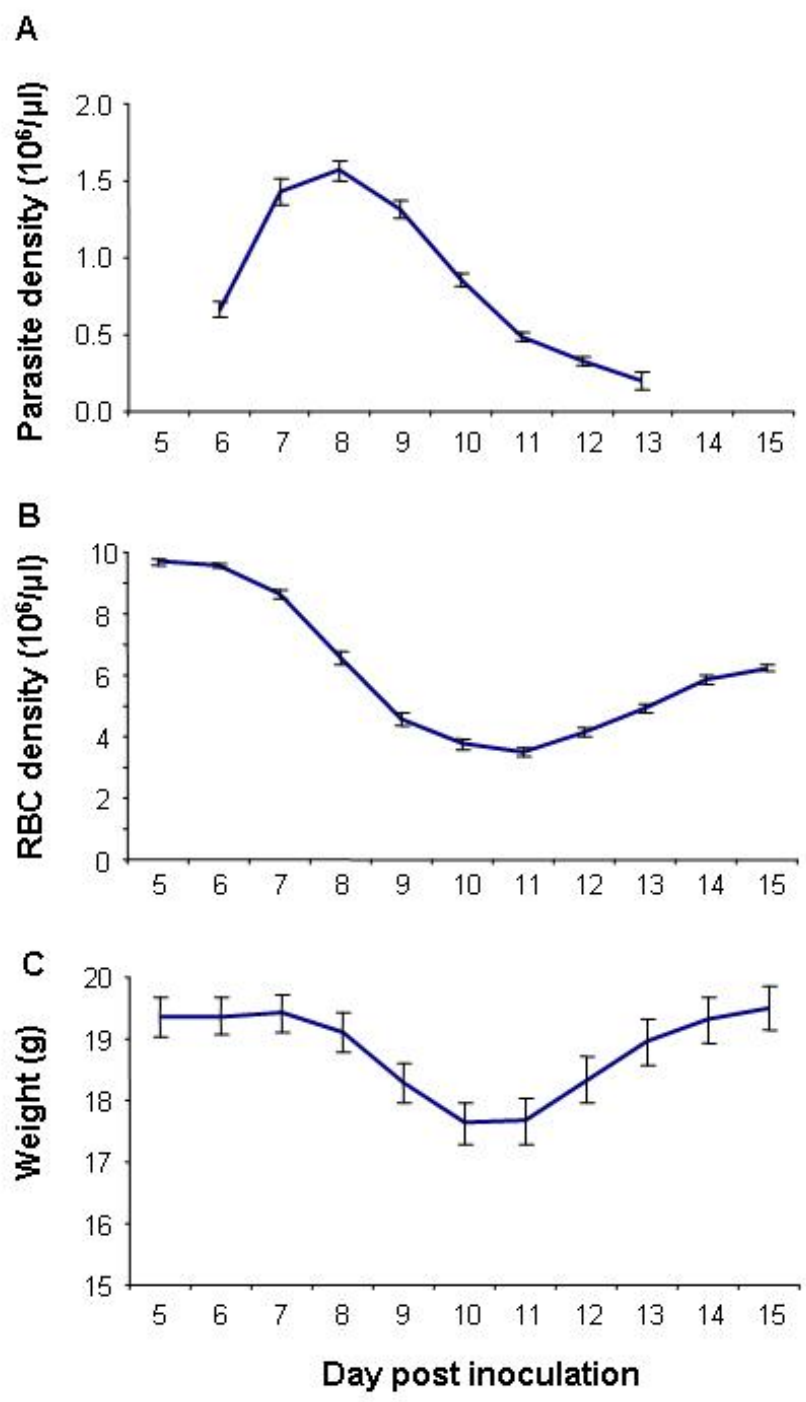

Fig 1S. Dynamics of infection across all mouse strains and parasite clones.

(A) Parasite (meants.e.) density over time. (B) RBC (meants.e.) density over time. (C) Weight (meants.e.) over time. 